\title{
PERFILES CONCEPTUALES COMO INSTRUMENTOS DE EVALUACIÓN DE UNA INTERVENCIÓN DIDÁCTICA
}

\section{Conceptual profiles as instruments for evaluating a didactic intervention}

\author{
Claudio Enrique \\ UDB Física - Departamento de Materias Básicas \\ Facultad Regional Santa Fe \\ Universidad Tecnológica Nacional, Argentina \\ cenrique@frsf.utn.edu.ar \\ Marta Yanitelli \\ Departamento de Física y Química \\ Facultad de Ciencias Exactas, Ingeniería y Agrimensura \\ Universidad Nacional de Rosario, Argentina \\ Silvia Giorgi \\ Departamento de Física \\ Facultad de Ingeniería Química \\ Universidad Nacional del Litoral, Argentina
}

Recibido 22 de mayo de 2019

Aceptado 19 de julio de 2019

\begin{abstract}
Resumen
Se exhiben los primeros resultados globales de una investigación, pertenecientes a una tesis de doctorado en la que se asume que mediante el empleo de Tecnologías de la Información y Comunicación, los estudiantes de ingeniería podrían modificar sus Perfiles Conceptuales sobre sistemas oscilantes. La idea de Perfil Conceptual de Mortimer reconoce que en un mismo sujeto conviven varios significados para la misma palabra o concepto -incluso para los de carácter científico- a los que se puede acceder en el contexto apropiado. En esta investigación, los perfiles fueron elaborados mediante una Matriz Epistemológica que incluyó tres dominios, y a partir de los niveles de comprensión, fueron propuestas cuatro zonas. En el cuestionario usado como instrumento se incorporó una consigna al final de cada pregunta donde el alumno debía indicar el grado de confianza con el que fueron dadas sus respuestas, lo cual fue usado para construir el Perfil Conceptual Relativo a la meta-cognición. Las actividades mediadas por Tecnologías de la Información y Comunicación se emplearon en la clase introductoria sobre Movimiento Oscilatorio, y en actividades experimentales con Laboratorios Virtuales de Aprendizaje. Los resultados mostraron que los estudiantes presentaron distintos niveles de compresión. Previamente a la intervención didáctica la mayoría se ubicó en la zona del Realismo interpretativo, mientras que a posteriori de ésta, la zona con más individuos correspondió al Racionalismo clásico. Respecto a los Perfiles Conceptuales Relativos a la meta-cognición, se observaron distintas situaciones donde los sujetos modificaron tanto su zona como su grado de confianza, observándose en general una evolución en la mayoría de los alumnos. En cualquiera de los casos, los Perfiles Conceptuales demostraron ser una herramienta novedosa e interesante para evaluar la intervención didáctica.
\end{abstract}

Palabras clave: Perfiles Conceptuales. Sistemas oscilantes. Mecánica clásica. Ingenierías.

\section{Abstract}

The first global results of a research are exhibited, belonging to a $\mathrm{PhD}$ thesis in which it is assumed that through the use of Information and Communication Technologies, engineering students could modify their Conceptual Profiles on oscillating systems. The idea of Conceptual 
Profile of Mortimer recognizes that in the same subject several meanings coexist for the same word or concept - even for those of a scientific nature -, which can be accessed in the appropriate context. In this investigation, the profiles were elaborated by means of an Epistemological Matrix that included three domains, and from the comprehension levels, four zones were proposed. In the questionnaire used as an instrument, a slogan was added at the end of each question where students should indicate the degree of confidence with which their answers were given, which was used to construct the Conceptual Profile related to metacognition. The activities mediated by the Information and Communication Technologies were used in the introductory class on Oscillation Movement, and in experimental activities with Virtual Learning Laboratories. The results showed that the students presented different levels of compression. Prior to the didactic intervention the majority was located in the area of interpretative realism, while after this, the area with more individuals corresponded to classical Rationalism. Regarding the Conceptual Profiles related to metacognition, different situations were observed where the subjects modified both their area and their degree of confidence, observing in general an evolution in most of the students. In any of the cases, the Conceptual Profiles proved to be a novel and interesting tool to evaluate the didactic intervention.

Keywords: Conceptual Profiles. Oscillating systems. Classic mechanics. Engineering. 


\section{Introducción}

El presente trabajo forma parte de una tesis de doctorado titulada "Integración conceptual de los movimientos oscilatorios armónico y amortiguado a través de actividades problemáticas significativas mediadas por TIC en el nivel universitario básico: diseño, desarrollo y evaluación”, en la que se asume como supuesto de partida que a partir del empleo de TIC los alumnos podrían modificar sus Perfiles Conceptuales (PC)(Mortimer\&Caravalho, 1994; Mortimer, 1995) cuando noten diferencias entre sus saberes y lo que el contexto externo le presenta, hasta adoptar un perfil que los satisfaga.

Por lo tanto, se consideró importante detectar los saberes del alumno previo a la enseñanza; a los cuales se asocian los denominados en este trabajo como Perfiles Conceptuales Iniciales (PCI).Junto a éstos se presenta una nueva versión, los Perfiles Conceptuales Iniciales Relativos (PCIR) a la meta-cognición de los estudiantes. Asimismo, luego de la implementación de una intervención didáctica mediada por TIC, diseñada específicamente para favorecer un aprendizaje significativo de los sistemas oscilantes, se indagó sobre los Perfiles Conceptuales Finales (PCF) y los Perfiles Conceptuales Finales Relativos (PCFR). La comparación entre éstos y los primeros permitió evaluar la influencia de dicha intervención en la comprensión de los conceptos científicos asociados a los movimientos oscilatorios armónico y amortiguado.

Cabe destacar que de acuerdo a la información disponible hasta el presente, no se registran investigaciones sobre PC en alumnos del ciclo inicial universitario relacionados con sistemas oscilantes.

\section{Referentes Teóricos}

Esta investigación tiene como marco referencial la teoría de los Perfiles Conceptuales de Mortimer, la cual se dio a conocer inicialmente en la tesis doctoral de dicho investigador en el año 1994. Según El-Hani\&Mortimer (2010), "los perfiles conceptuales constituyen, precisamente, modelos de diferentes maneras de ver y representar el mundo que son utilizadas por las personas para significar su experiencia" (pp. 327). Es decir, la idea de PC presupone que en un mismo sujeto se da la convivencia de dos o más significados para la misma palabra o concepto - incluso dentro de los conceptos científicos - , a los que se puede acceder en el contexto apropiado. La noción de PC (Mortimer, 1995) considera que cada una de las diferentes representaciones de un sujeto establece una zona de PC, que está constituida por elementos epistemológicos y ontológicos que se utilizan dependiendo del contexto. Además, considera que dichos PC están íntimamente relacionados con sus ideas o conocimientos previos. De este modo, cualquier individuo puede poseer más de una forma de comprensión de un determinado concepto; es decir, diferentes zonas de un PC pueden convivir en el mismo individuo, que corresponden a distintas formas de pensar y hablar, donde "la intención es construir un modelo para describir la evolución de las ideas tanto en el espacio social de la clase como en los individuos, a consecuencia del proceso de aprendizaje" (Mortimer, 2000, pp. 53).

Para evaluar los PC, debe citarse que "el conocimiento y el aprendizaje están situados, es decir, la cognición es parte de la actividad, el contexto y la cultura en la cual se desarrolla y en la que va a ser utilizada" (Solsona et al., 2001). Según estos mismos autores, 
los niveles consecutivos del perfil conceptual reflejan la habilidad del alumnado para dar coherencia y significado a la diversidad de hechos que encuentra a su alrededor, tanto en la clase... como fuera de la misma. Estos perfiles conceptuales serán más operativos y correctos, a medida que el alumnado sea más competente en la interpretación de fenómenos, identificando las propiedades... y manejando los... niveles de explicación... (pp. 3).

Dicho de otro modo, “su desarrollo cognitivo será descrito en función de los diferentes tipos de perfiles conceptuales" (pp.3).

Por su parte, Viggiano \& Rodrigues de Matto (2007) indicaron que las investigaciones de Mortimer dejan en segundo plano el contexto, una de las principales contribuciones de la noción de PC. Por ello, aducen que éste debe ser considerado al diseñar instrumentos para la construcción de PC, dado que es importante en la evolución de los mismos, además de incluir nuevas zonas, o la modificación de las preexistentes. En esta investigación, uno de los propósitos es incorporar la metacognición, dado que la misma se refiere al conocimiento que tienen los estudiantes sobre los propios procesos cognitivos o sobre cualquier elemento relacionado con ellos; es decir, las propiedades de la información o los datos relevantes para el aprendizaje, y, entre otras cosas, el control y la regulación subsiguiente de estos procesos (Flavell, 1976).

\section{Metodología}

El grupo que participó en esta indagación estuvo conformado por 44 estudiantes regulares (comisión "A" cohorte 2017) de la materia Física I, perteneciente al primer año de la carrera de Ingeniería Civil que se dicta en la Universidad Tecnológica Nacional - Facultad Regional Santa Fe, Argentina, quiénes participaron en todas las actividades diseñadas en el marco de la tesis: pre-test; intervenciones didácticas mediadas por TIC y pos-test. Además, estuvieron presentes en las tareas propias del dictado de la materia Física I respecto a los contenidos asociados a sistemas oscilantes.

La secuencia de acciones llevadas a cabo y los instrumentos para la obtención de datos pueden sintetizarse de la siguiente forma:

- Administración de un cuestionario como pre-test para el relevamiento de conocimientos previos.

- Desarrollo de la unidad didáctica sobre movimientos oscilatorios armónico y amortiguado, consistente en la integración intencionada de experimentos en tiempo real y simulaciones.

- Diseño de la Matriz Epistemológica para construir las zonas de PC.

- Administración, como pos-test, del mismo cuestionario utilizado como pre-test.

- Análisis estadístico - tanto descriptivo como inferencial - para muestras relacionadas a fin de detectar si hubo diferencias significativas entre los PCI y PCF y los PCIR y PCFR.

\section{Pre-test}

Como instrumento de obtención y registro de información, sobre los conocimientos previos que disponen los estudiantes, se elaboró un cuestionario 
(Enrique, Yanitelli\&Giorgi, 2018) que tuvo como referentes varias investigaciones preliminares sobre Movimiento Oscilatorio, entre las que se destacan las de Clement (1982); Viennot (1989); Santos Benito et al. (2005); y García Barneto\& Bolívar Raya (2005). En particular sobre las investigaciones a nivel local en la UTN - FRSF se puede citar el trabajo de Enrique (2017).

Con el objeto de contextualizar los contenidos teóricos a abordar en una situación real, en el cuestionario se presentó una introducción con una narrativa sobre un personaje de historietas que está jugando en una hamaca; y se indicó que al analizar el movimiento de ida y vuelta desde el punto de vista de la Mecánica Clásica, se llegaría a la conclusión de que el movimiento de la hamaca es oscilatorio. Posteriormente, se establecieron las condiciones para modelizar el sistema como un péndulo simple y se orientó el análisis del movimiento desde un marco de referencia inercial o fijo en la Tierra. Finalmente se presentaron las 12 preguntas que conformaron el cuestionario, siendo las mismas abiertas y cerradas de opción múltiple. Al término de cada una de ellas, con el objeto de incorporar a la meta-cognición en la indagación, se agregó una consigna complementaria destinada a calificar el grado de confianza ante la respuesta dada, cuyo intervalo va desde 0 -confianza nula- a 10 -confianza plena-. Si bien tal consigna ya fue presentada en otras investigaciones (García Barneto\& Bolívar Raya, 2008), no se ha realizado un análisis detallado de la misma.

Para validar dicho instrumento, fue sometido a las pruebas estadísticas de validez (Prueba de KMO y de Esfericidad de Bartlett) y de fiabilidad (Alfa de Cronbach) las que permitieron, además, seleccionar las categorías conceptuales para el análisis de las respuestas.

\section{La Unidad Didáctica}

Sólo a título informativo, se describe el diseño de la Unidad Didáctica donde se hace uso de TIC como mediadoras delos procesos de enseñanza y de aprendizaje.

La propuesta didáctica tiene como objetivo el estudio de los Movimientos Oscilatorios Armónico Simple (MAS) y Amortiguado (MOA). Específicamente, se incluyen actividades mediadas por TIC en las que se emplean dispositivos con toma de datos en tiempo real para indagar acerca de la evolución temporal de distintas magnitudes físicas- Fuerza neta; posición, velocidad y aceleración angular que caracterizan a dichos movimientos - y Laboratorios Virtuales de Aprendizaje (LVA) que consisten en simulaciones seleccionadas con formato en JAVA.

El estudio de los sistemas oscilantes - descrito tradicionalmente mediante el uso de péndulos - tiene gran importancia en el estudio de las Ciencias Experimentales y en particular en la Física. El MAS es uno de los movimientos idealizados más estudiado, pues constituye una buena aproximación a muchas de las oscilaciones que se dan en la naturaleza y es "sencillo" de describir matemáticamente (Alonso y Finn, 1995). Además, es un tema relevante para los futuros ingenieros, dadas sus múltiples aplicaciones como por ejemplo, en Ingeniería Civil, en "el análisis de estructuras sometidas a cargas dinámicas como pueden ser las que aparecen durante un sismo, acción del viento, explosiones u otros eventos accidentales" (Brotons et al., 2014, pp.187).

Las actividades de la intervención didáctica comprenden tres fases: 
a. Iniciación. Corresponde a una fase introductoria, previa al inicio de la clase de teoría, en la que se trabaja con un sistema de toma de datos en tiempo real que permite analizar el movimiento de un péndulo simple y de uno físico.

b Desarrollo. Comprende las clases tradicionales de teoría, resolución de problemas, y un trabajo práctico - péndulo simple -.

c. Aplicación. Incluye la realización de experiencias en LVA conformado por simulaciones en JAVA con los péndulos simple y de resorte, tanto para el MAS como para el MOA.

La primera fase cumple con el objetivo de "mostraren una pantalla” a los estudiantes el comportamiento de un sistema bajo la acción de una fuerza variable en el tiempo, y que al ser periódica en el corto plazo, se puede asociar a una función senoidal o cosenoidal, Figura 1.

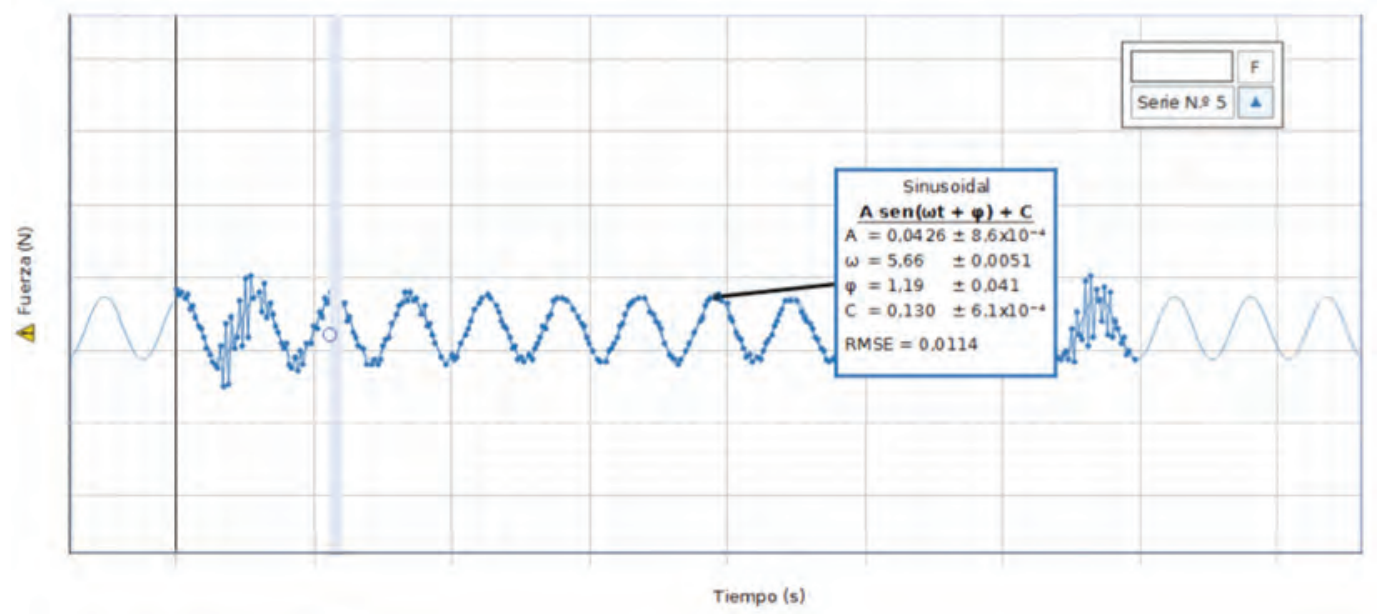

Figura 1: Fuerza neta (N) en función del tiempo (s) de un péndulo simple que realiza un MAS.

Por otro lado, al efectuar un análisis cinemático del movimiento de un péndulo físico, presentando en una pantalla las variaciones simultáneas de la posición, velocidad, y aceleración angulares en función del tiempo, se observa la presencia de un sistema amortiguado. Pero si se disminuye el tiempo de análisis se percibe un sistema armónico, En particular para la aceleración angular, se obtuvo la Figura 2.
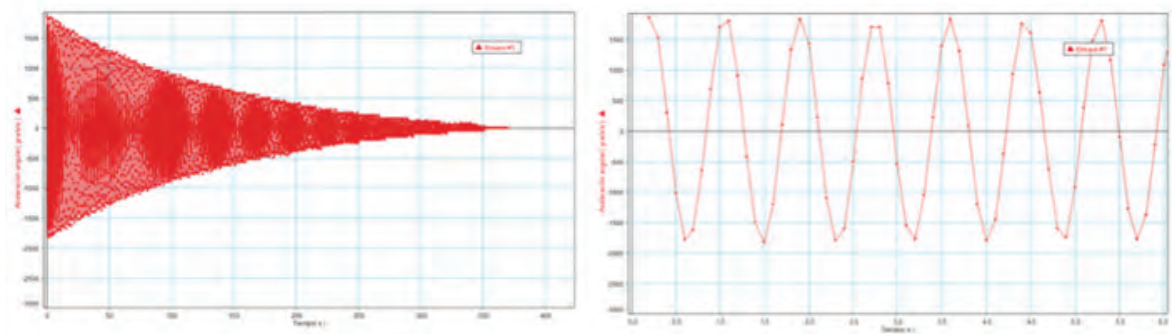

Figura 2: Aceleración angular $\left(\mathrm{rad} / \mathrm{s}^{2}\right)$ en función del tiempo (s) para una oscilación amortiguada -para tiempos largos -(izquierda) y para una armónica- para tiempos cortos -(derecha).

Luego de las clases tradicionales de teoría, de problemas - incluyendo la resolución en grupos y su presentación en clase-y de un trabajo prácticorealizado con péndulo simple en el aula, se continuó con la realización de diferentes experiencias mediante LVA. Los mismos fueron seleccionados teniendo en cuenta tanto la confiabilidad como la validez de las fuentes de dichos programas. En 
esta actividad se propone que las tareas se realicen por grupos de estudiantes, con el objeto de favorecer el aprendizaje colaborativo; las actividades consisten en hacer uso de varias simulaciones con péndulos simple y de resorte, tanto para un MAS como un MOA. Se diseñaron tres experiencias con LVA:

a. Análisis integral de los péndulos simple y de resorte.

b. Análisis cinemático de un péndulo de resorte.

c. Oscilaciones armónicas y amortiguadas.

En base a la gran oferta que existe en internet sobre applets de sistemas oscilantes armónicos y amortiguados, se seleccionaron cuatro simuladores en JAVA de acceso libre de acuerdo a las características citadas anteriormente. Las capturas de pantallas de los mismos se presentan en la Figura 3.

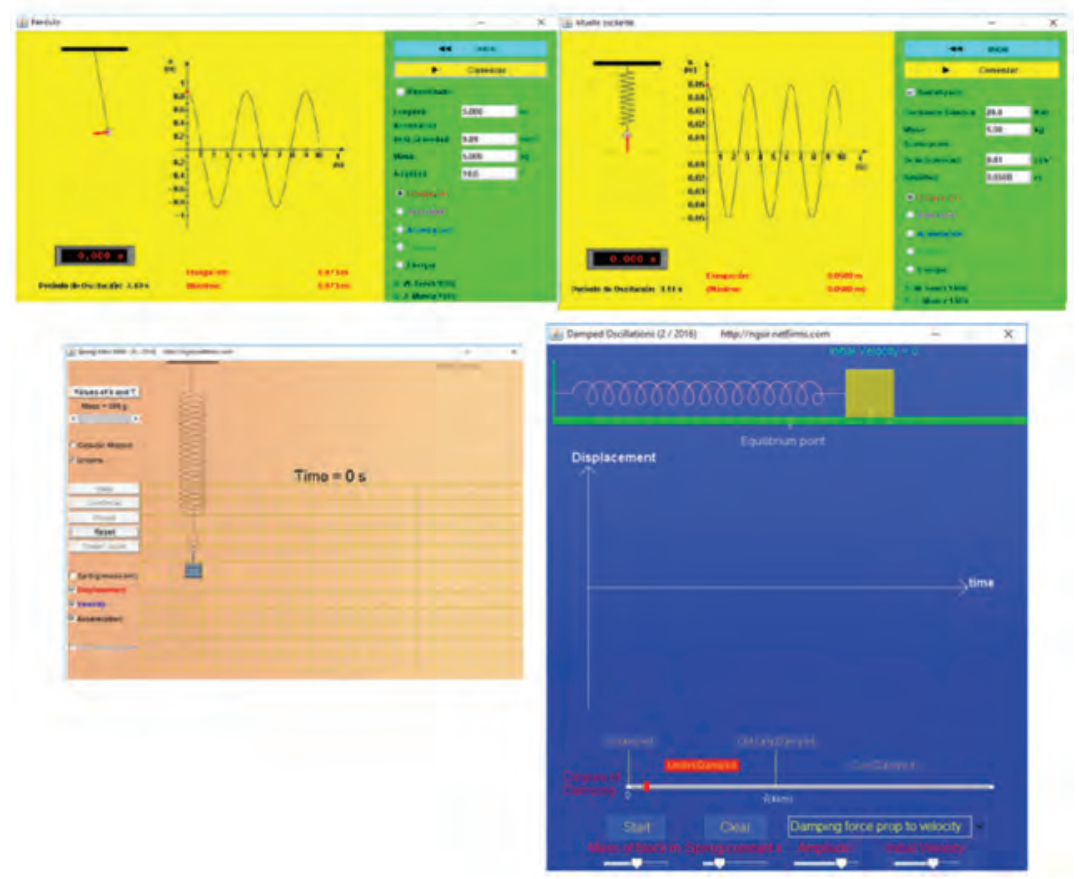

Figura 3: Captura de pantallas de los Appletsen Java: péndulos simple y de resorte para el estudio del MAS (arriba y abajo izquierda) y del MOA (abajo derecha).

Fuente: https://www.walter-fendt.de/html5/phes/ ; https://ngsir.netfirms.com/

\section{Matriz Epistemológica}

Para la construcción de los PC, tanto iniciales como finales, se diseñó una Matriz Epistemológica (Enrique, Yanitelli\&Giorgi, 2019). En ella se consideraron tres ámbitos genéticos: el sociocultural, el ontogenético y el micro-genético. El primero de ellos, también denominado socio-histórico, involucra el estudio de los sistemas oscilantes en la historia del conocimiento científico. Como en el estudio de estos sistemas no existe una categoría propia asociada a sus conceptos -como en el caso de la masa o la fuerza-, se empleó el estudio del movimiento en la historia de la Física. En el dominio ontogenético se tomaron como referentes varias investigaciones publicadas acerca de ideas previas sobre sistemas oscilatorios. Dentro del último dominio se encuentran los resultados del pre-test obtenidos a partir del análisis de las respuestas al cuestionario. A continuación, de manera resumida, se indican las características de los tres dominios. 
Con respecto del dominio socio histórico, se definieron tres momentos históricos: a. Aristotélico; b. Medieval o del Ímpetu; y c. Mecánica Clásica o Newtoniana.

Se da inicio con la Física aristotélica como teoría "pre-científica" del movimiento, la cual adopta principios metafísicos o trascendentales, debido a que la Física de Aristóteles, así como muchas de las ideas propuestas por los antiguos filósofos acerca del movimiento, "en la actualidad están bastante presentes en la manera de pensar de las personas inexpertas en Física” (Camargo et al, 2007, pp. 172). Para Cohen (1967), la física aristotélica es conocida a veces como la física del sentido común, porque es en la que la mayoría de las personas creen y por la cual se guían intuitivamente, o que parece interesar y agradar a cualquier individuo que use su inteligencia natural pero que no haya aprendido los modernos principios de la dinámica. Según Piaget \& García (1982), otro punto importante para la comprensión de la doctrina aristotélica del movimiento es la necesidad de recurrir a un "motor" como la causa de dicho movimiento. Además, investigaciones en el área de las concepciones espontáneas han demostrado que la Física de sentido común mantiene estrechas relaciones con la física aristotélica y/o con el pensamiento medieval del ímpetu (De Lozano et al, 2016; Herrera Espinosa, 2013; Mora \& Herrera, 2009; Mora \& Benítez, 2007; Pozo, 1987).

El pensamiento medieval está incluido en una etapa intermedia entre las concepciones aristotélicas y las aceptadas por la Mecánica Clásica. Dentro de éstas, la teoría del ímpetu de Buridan (1300 - 1358), en la que su idea de fuerza motriz se puede explicar a partir del movimiento de un proyectil: después de dejar el motor (lanzador), el proyectil sería movido por un "ímpetus"- es decir, por una especie de poder de auto-movimiento - capaz de moverlo en la dirección dada por el motor (López, 2009). Así llegó a la conclusión de que el motor produce una "virtus motiva" en el móvil. El movimiento incluso podría seguir infinitamente, pero no era posible por las influencias externas como la resistencia del aire y la "gravedad". Dicho de otro modo, el "ímpetus" se debilitaría continuamente hasta que la gravedad predominara y el proyectil retornase a su lugar natural. Esta teoría se caracterizó por tres propiedades:

- cuanto mayor es la velocidad con la que el motor mueve el cuerpo, mayor será el ímpetu impreso en el cuerpo en movimiento;

- cuanto mayor es la materia del cuerpo movido, más intenso será el ímpetu recibido por él;

- la tercera está asociada a la noción primitiva de inercia: el ímpetu es cualquier cosa cuya naturaleza es permanente y no es "corrompido" por la resistencia que le ofrece el medio.

Por otro lado, Avicena (980 - 1037) (citado en Peduzzi\&Zylbersztajn, 1997) propuso que en el caso del movimiento de un proyectil lanzado horizontalmente, éste se mueve inicialmente en línea recta - en la dirección en que fue lanzado -, y continúa su movimiento horizontal hasta que la fuerza (horizontal) que le fue impresa sea totalmente agotada. Cuando eso ocurre, el proyectil se detiene, momentáneamente, y luego se mueve hacia abajo, bajo la acción de su "peso natural". Expresado de otro modo, la trayectoria del proyectil es la de una "L" invertida.

En el estudio del dominio ontogenético, se consideraron distintas investigaciones sobre las ideas previas de los estudiantes acerca de los sistemas oscilantes. Desde las primeras observaciones de Viennot (1979) hasta las recopilaciones más actuales, se ha producido una gran cantidad de conocimientos en este campo. 
Sin embargo, no abundan las referencias a los conocimientos previos que los estudiantes manifiestan tanto sobre el MAS como el MOA. Los ensayos reportados indican que, a pesar de que el péndulo simple es un modelo recurrente en la mayoría de los textos de Física para enseñar cuestiones relacionadas con Mecánica, los estudiantes muestran dificultades en su comprensión (Manabu, 2004; Czudkova \& Musilova, 2000).

En relación al dominio micro-genético se tomaron en cuenta las ideas que derivan de las respuestas al pre-test. En el análisis de dichas respuestas se consideraron las siguientes categorías conceptuales: Conceptos básicos; Cinemática; Dinámica; Energía; Conservación y No conservación.

\section{Análisis Estadístico}

Con el objeto de cuantificar si hubo diferencias entre los PCI y PCF Globales, se realizó un análisis estadístico - tanto descriptivo como inferencial - para muestras relacionadas. Debe aclararse que como uno de los estudiantes no completó el cuestionario del pos-test con el grado de confianza a cada respuesta, el análisis comparado incluye a 44 estudiantes. Además, en el análisis estadístico se incluye la opción "exactas", dado que el número de sujetos es menor a 50.

La prueba de rangos con signos de Wilcoxon es una prueba de contrastes no paramétrico, donde se tiene en cuenta tanto el signo como la magnitud de las diferencias entre los valores de cada par; en este caso, PCI versus PCF, y grado de confianza inicial versus final. Además se completó con la prueba de los signos, la cual calcula las diferencias entre las dos variables para todos los casos, clasificándolas como positivas, negativas, o empatadas. Cuando las dos variables testeadas tienen la misma distribución, el número de las diferencias tanto positivas como negativas son similares. De todos modos, la prueba de Wilcoxon suele ser más potente que la de los signos. Finalmente, se hace el estudio de la significación, es decir, si se acepta la Hipótesis Nula $\left(\mathrm{H}_{0}\right)$, que contempla la igualdad entre las sumas de los rangos de las diferencias positivas $[\mathrm{S}(+)$ ] y la suma de los rangos de las diferencias negativas [S (-)]; mientras que la Hipótesis Alternativa $\left(\mathrm{H}_{1}\right)$ no lo considera:

$$
\begin{aligned}
& \mathrm{H}_{0}: \mathrm{S}(+)=\mathrm{S}(-) \\
& \mathrm{H}_{1}: \mathrm{S}(+) \neq \mathrm{S}(-)
\end{aligned}
$$

\section{Resultados y discusión}

El análisis de las producciones de los estudiantes al responder el pre-test muestra que sus saberes previos están comprendidos entre las tres categorías del dominio socio histórico presentadas previamente; a pesar de que ya habían desarrollado contenidos de Mecánica Clásica asociados al movimiento de la partícula y del sólido rígido.

A continuación se presenta un caso representativo, identificado como alumno 42, en el que sus respuestas evidencian concepciones cercanas a la física aristotélica. En este sentido se observó que en la Pregunta 11, corrige la opción C - que es la correcta - por la A, Figura 4, denotando que percibe que se trata de una caída libre con componente horizontal nula de la velocidad inicial. 
11. Una esfera suspendida de un hilo se mueve pendularmente como lo indica la Figura $5 \mathrm{c}$

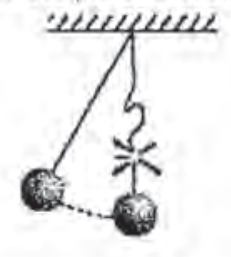

Figura 5

Guando pasa por su punto más bajo, el hilo se corta La trayectoria descrita por la esfera es la mostrada en:

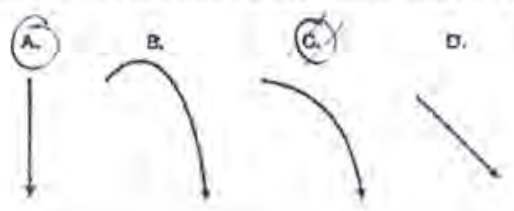

A. Marca con un circulo la opción que consideres adecuada.

B. Grafica el vector velocidad sobre la esfera un instante antes de que el hilo se corte.

Indica el grado de confianza que tienes en la respuesta dada (de 0 - confianza nula - a 10 - confianza plena -) is

Figura 4: Respuesta del alumno 42 en la pregunta 11 del pre-test.

Y, en la misma pregunta, acompañó la gráfica del vector velocidad con la justificación que se presenta en la Figura 5:

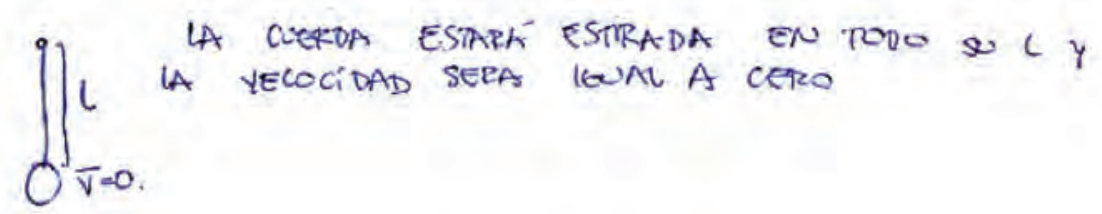

Figura 5: Justificación del alumno 42 en la pregunta 11.b del pre-test.

En esta proposición se observa una concepción aristotélica, donde se considera que "los cuerpos que se mueven poseen fuerzas, que no son meras acciones externas sino que se quedan en el objeto y van consumiéndose" (Gil Pérez \& Carrascosa Alís, 1992, pp. 319).

Esta concepción, consistente en que el sistema (hamaca + asiento) está en reposo cuando pasa por la posición $\mathrm{C}$ de equilibrio, ya la había manifestado en Pregunta 1 , al construir el diagrama de cuerpo libre (DCL)del sistema modelizado como un péndulo simple, Figura 6.

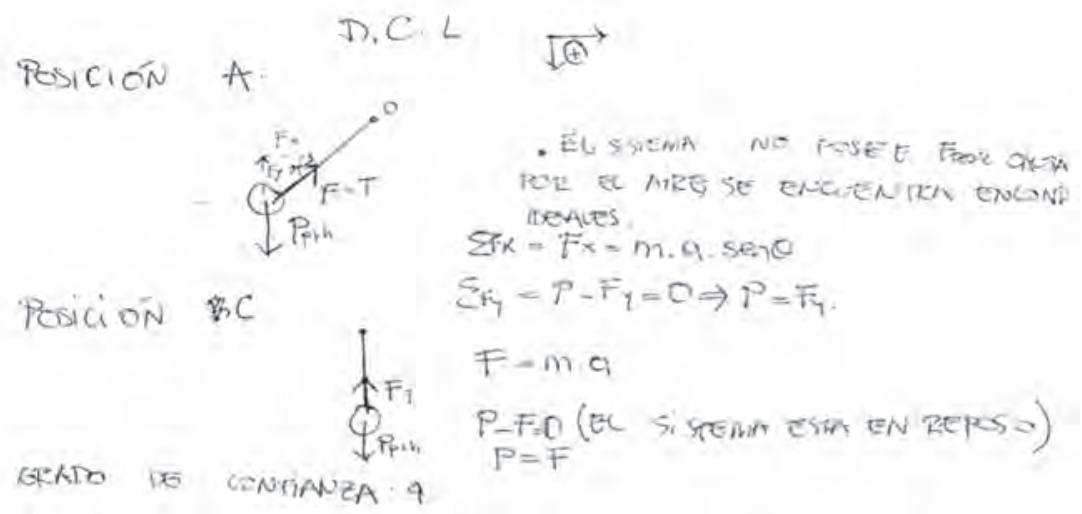

Figura 6: DCL elaborados por el alumno 42 en la pregunta 1 del pre-test. 
Al proponer que en la posición de equilibrio "el sistema está en reposo", confunde "posición de equilibrio" con "condición de equilibrio", en la que la aceleración tangencial es nula.

Por otro lado, como la mayoría de los estudiantes, no optó por asociar un sistema conveniente de ejes coincidentes con las direcciones tangencial y radial para el análisis dinámico del movimiento en un tiempo dado; sino que apeló al uso del clásico fijo a un cuadrante que implica trabajar con las proyecciones del vector aceleración en ambos ejes. En la Figura 6 se observa que en el DCL efectuado para una de las posiciones extremas del péndulo simple, identificada como Posición A, solo consideró la proyección de la aceleración en el eje x.

Dentro de los casos que en la Pregunta 1 no logran modelizar el sistema como péndulo simple, el alumno 47 indicó, Figura 7, sin confianza en su respuesta:

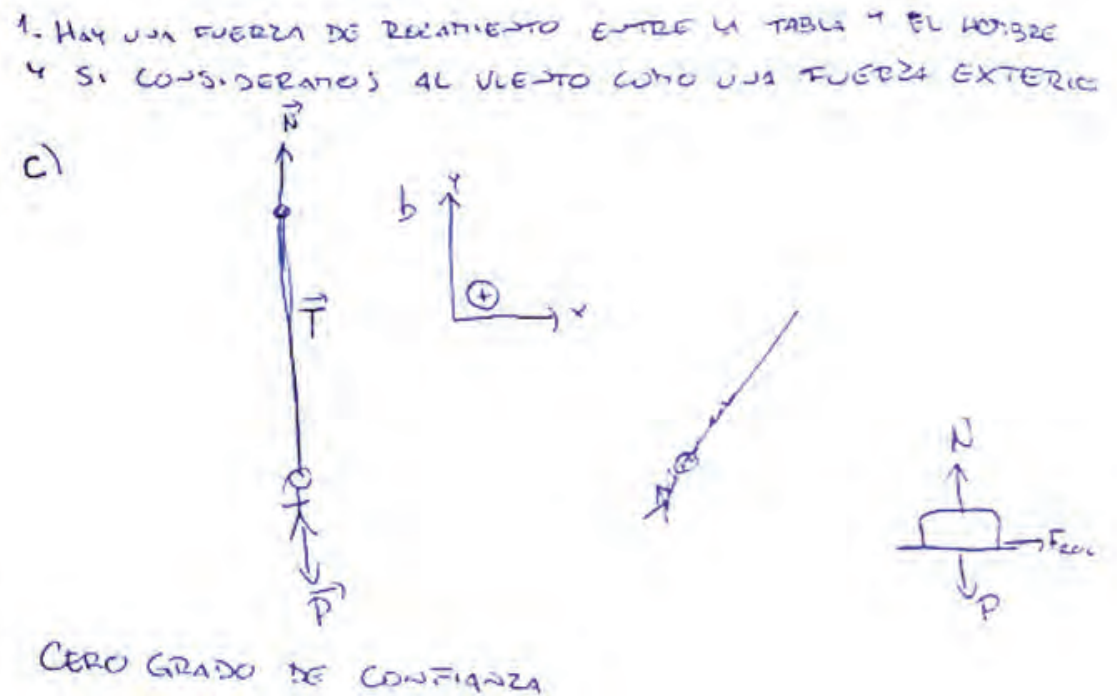

Figura 7: Respuestas del alumno 47 en la pregunta 1 del pre-test.

La proposición "Hay una fuerza de rozamiento entre la tabla y el hombre" pone en evidencia que, si bien la fuerza de rozamiento está presente, no ha internalizado que se trata de una fuerza interna. Además, se observa lo que se puede denominar una especie de "terror al vacío", ya que considera la presencia de un medio material, el viento, como necesario para justificar una "fuerza exterior". En la Pregunta 12 retoma su razonamiento. La respuesta se muestra en la Figura 8.

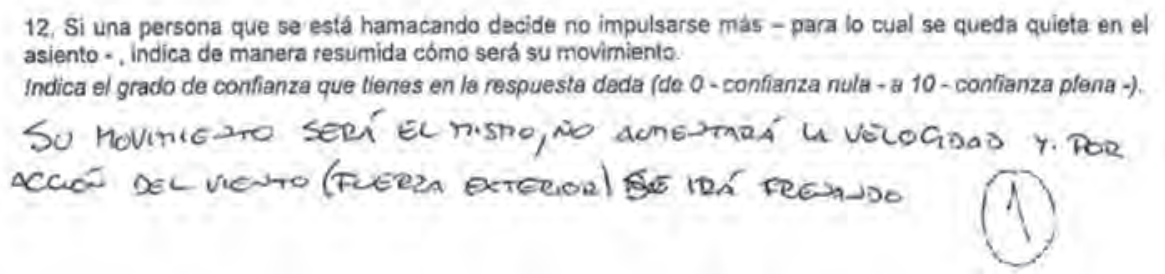

Figura 8: Respuesta del alumno 47 en la pregunta 12 del pre-test.

La concepción medieval que se ha destacado en los estudiantes es la presencia de una fuerza en la dirección del movimiento. Un caso representativo es el alumno 4 que, en la Pregunta 1, al construir el DCL del sistema (hamaca + asiento), modelizado comopéndulo simple, cuando pasa por la posición de equilibrio in- 
cluye una fuerza horizontal con sentido hacia la izquierda, Figura 9. Además, lo confirma al argumentar "ya que viene con un movimiento de igual dirección".

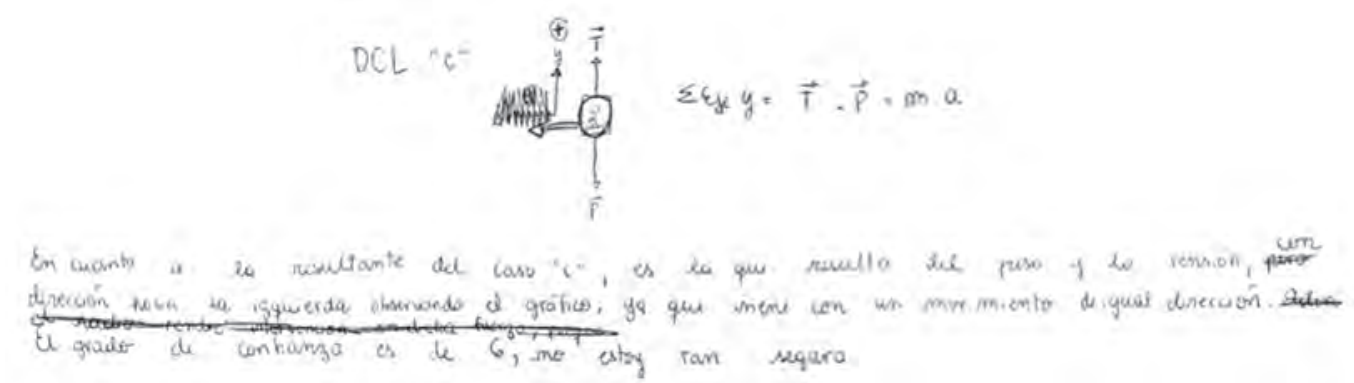

Figura 9: Explicaciones del alumno 4 en la pregunta 1 del pre-test.

Otro caso, identificado como alumno 26, explicita en la misma pregunta "Al haber un empuje o fuerza exterior $(\mathrm{Fe})$ que permite el movimiento oscilatorio debe haber una fuerza que sea tangencial a la circunferencia generada por el movimiento de la hamaca, que se hace nula por un instante en las posiciones extremas". En la última parte de su argumentación se evidencia que considera que cuando el sistema llega a las posiciones extremas la fuerza exterior es igual a cero, asociándola a la velocidad instantánea que sí es nula; es decir, percibe una fuerza proporcional a la velocidad.

Dentro de la concepción Mecánica Newtoniana, los resultados más destacados estuvieron asociados a la identificación de la periodicidad de la fuerza resultante y las formas de energías presentes en las posiciones extremas y de equilibrio. En el caso del alumno 25 se observa, Figura 10, que no sólo identifica la periodicidad de la fuerza neta en dos períodos al responder la Pregunta 2, sino que además identifica las posiciones instantáneas indicadas en las consignas del cuestionario con A y B las que corresponden a las posiciones extremas del péndulo simple y con $\mathrm{C}$ a la posición de equilibrio.

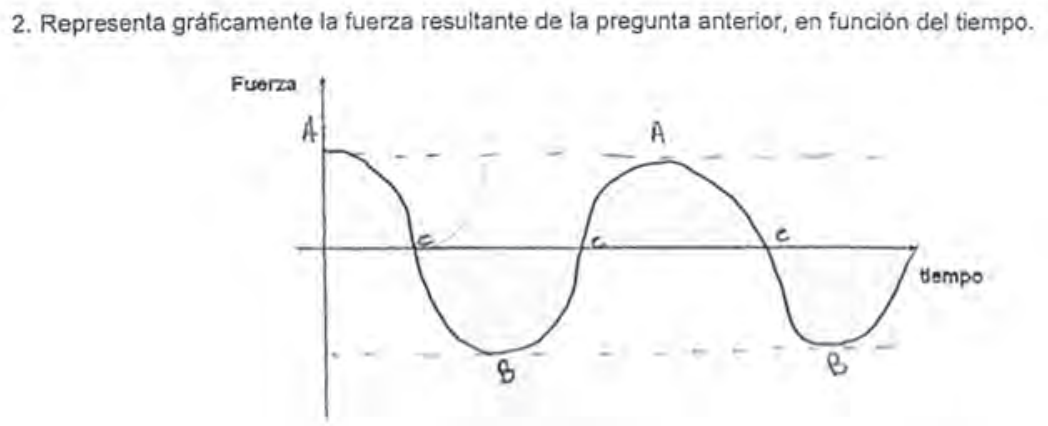

Figura 10: Producción del alumno 25 en la pregunta 2 del pre-test.

El alumno 9 respondió la Pregunta 3 mediante un razonamiento correcto respecto de la energía del sistema, Figura 11. 


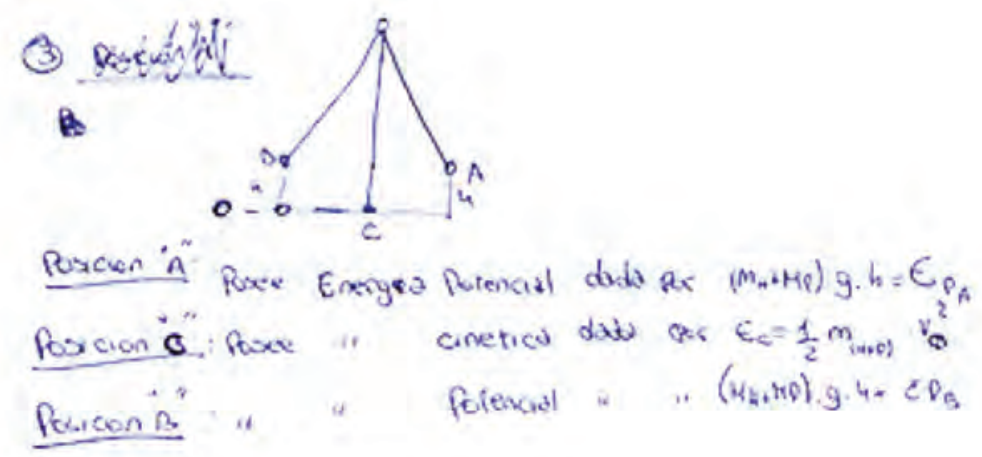

Figura 11: Análisis energético efectuado por el alumno 9.

Propone de manera adecuada el "0" de energía potencial, indica las formas de energías en cada una de las posiciones, y explicita las ecuaciones correspondientes.

Los resultados obtenidos a partir del análisis de las respuestas del pre-test, permitieron identificar cuatro zonas de PCI. A continuación se presenta, en forma sucinta, las principales ideas características de cada una de ellas.

- Zona 1. Realismo ingenuo. Está relacionada con los niveles de percepción inmediata y sensible de los fenómenos estudiados y corresponde a ideas intuitivas que justifican de manera muy limitada la ocurrencia natural de los mismos, por lo que no son consideradas las condiciones en que los procesos ocurren. Interpretan los $\mathrm{MO}$ de manera aleatoria y sin establecer relaciones en el análisis de los hechos, ni los relacionan entre sí mediante determinados procesos, o directamente no lo logran.

- Zona 2. Realismo interpretativo. Está vinculada con la proximidad a los fenómenos por medio de la investigación, siendo la experiencia traducida en términos de constructos de la ciencia, en la cual son enfatizadas condiciones físicas experimentales. Interpretan los sistemas oscilantes en base a estados, y los analizan a partir de datos o hechos, aunque desconectados entre sí.

- Zona 3. Racionalismo clásico. Alude a un plano teórico de comprensión donde el análisis de los procesos está asociado al uso de algoritmos y fórmulas matemáticas que derivan de la consideración de modelos científicos teóricos, sin que eso se traduzca en evidencia de la comprensión de las relaciones conceptuales. Interpretan los $\mathrm{MO}$ como una sucesión de hechos, relacionados entre sí mediante ciertos procesos.

- Zona 4. Racionalismo sistémico. Refiere, al analizar los procesos, a un plano teórico de comprensión asociado al uso de algoritmos y ecuaciones matemáticas provenientes de modelos científicos teóricos. El entendimiento de los MO se realiza mediante la activación de relaciones conceptuales y su integración pasando a conformar un "todo". Interpretan los MO a partir de relaciones complejas que forman parte de un sistema.

A posteriori de la intervención didáctica se realizó el pos-test empleando el mismo cuestionario que en el pre-test. Luego del análisis de las preguntas, y mediante la misma Matriz Epistemológica, se clasificaron las respuestas para obtener tanto el PCF, como así también el PCFR. En la Figura 12, se han representado mediante barras las zonas de menor a mayor carácter explicativo de PCI y PCF (Globales). La Zona 1 corresponde al Realismo ingenuo, la Zona 2 al Realismo interpretativo, la Zona 3 al Racionalismo clásico y la Zona 4 al Racio- 
nalismo sistémico. La altura de las barras muestra, en frecuencia, la distribución de los estudiantes asociados a cada zona.

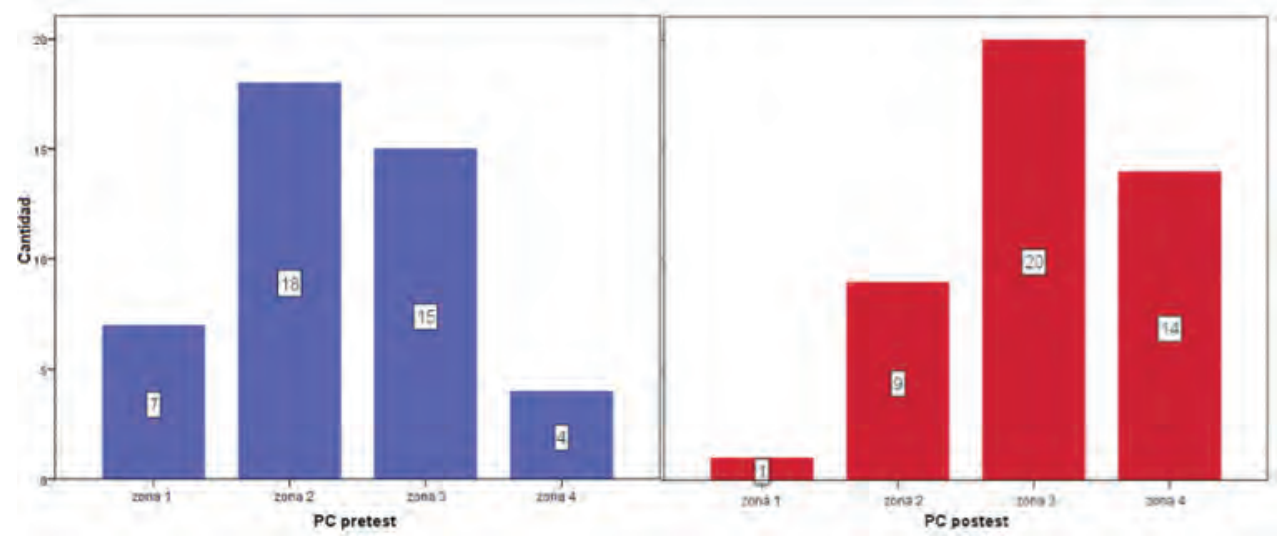

Figura 12: Zonas de PCI y PCF (Globales).

En cada barrase indica el número de estudiantes en cada zona de PCI o PC pre-test (barras de color azul) y PCF o PC pos-test(barras en color rojo) ambos Globales. Se observa una evolución debido a la intervención didáctica dado que en el PCI la Zona 2Realismo interpretativo es la más destacada mientras que en el PCF lo es la Zona 3 Racionalismo clásico; además, se evidenció una disminución del número de estudiantes en la Zona 1 Realismo ingenuo y un incremento en la Zona 4 Racionalismo sistémico.

También fue posible identificar a los estudiantes que pertenecen a cada zona. Así, los sujetos codificados con los números 45, 42, 37, 29, 22, 12 y 10 pertenecen a la Zona 1 Realismo ingenuo, Figura 13. Luego de la intervención didáctica, sólo el alumno 2 está en dicha zona. Los codificados como 29, 42 y 45, pasaron a la Zona 2 Realismo interpretativo, mientras que los identificados como 10, 12, 22 y 37, lo hicieron a la Zona 3 Racionalismo clásico, Figura 14.

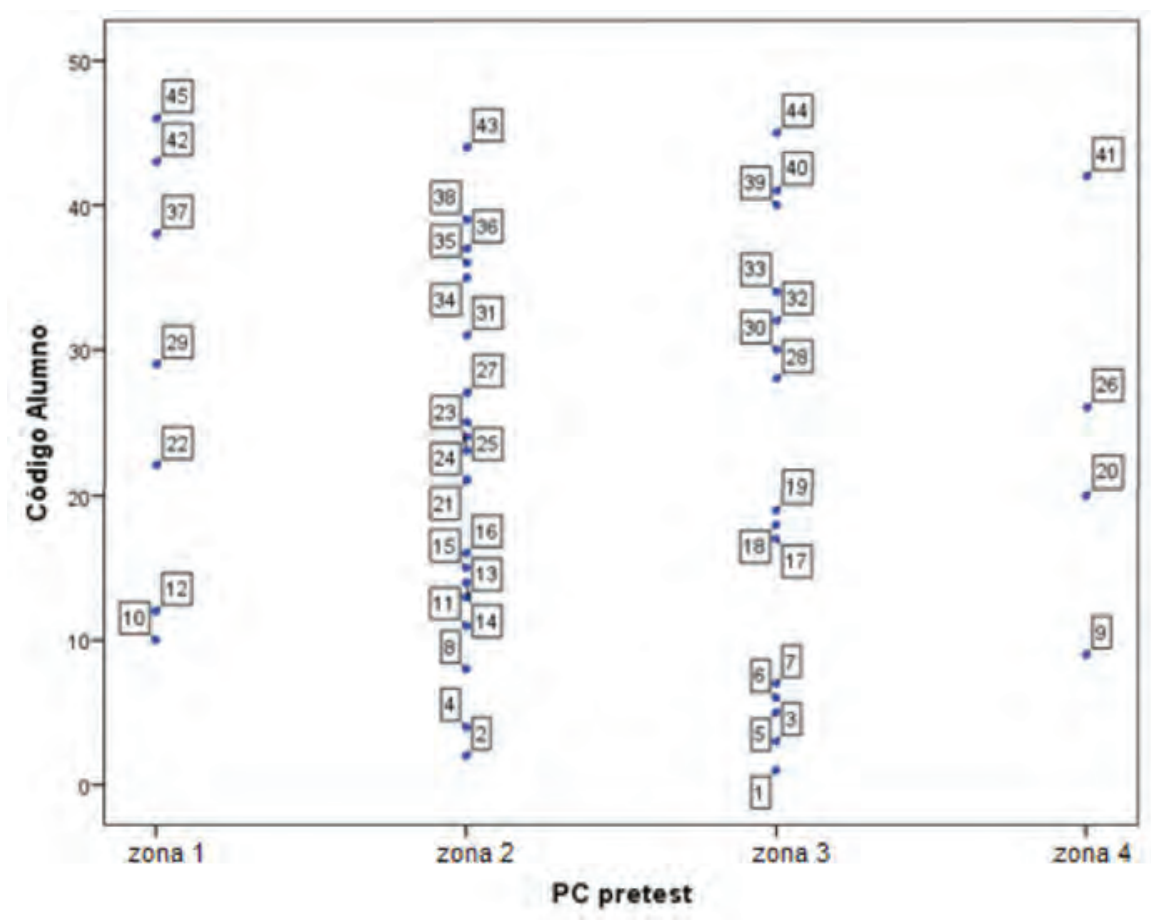

Figura 13: Ubicación de los alumnos de acuerdo a la Zona de PCI. 


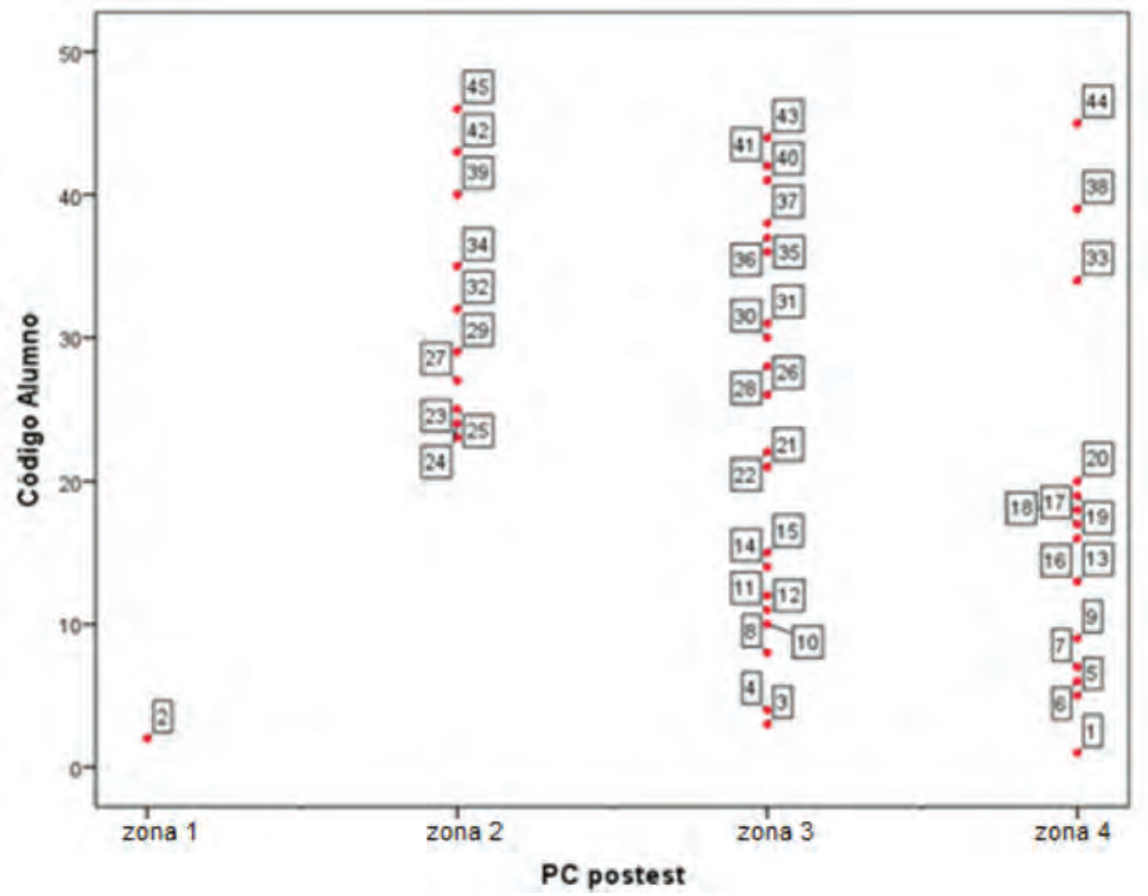

Figura 14: Ubicación de los alumnos de acuerdo a la Zona de PCF

Respecto al PCR, se puede ver que el alumno 22, que estaba en la Zona 1 Realismo ingenuo, Figura 15, y con un grado de confianza ( $\mathrm{G}$ de $\mathrm{C}$ pre-test) en sus respuestas al pre-test igual a 5, pasó a la Zona 3 Racionalismo clásico, Figura 16, con un grado de confianza ( $\mathrm{G}$ de $\mathrm{C}$ pos-test) 7 . Es decir, no sólo evolucionó en su nivel de comprensión, sino también en su confianza al responder el cuestionario.

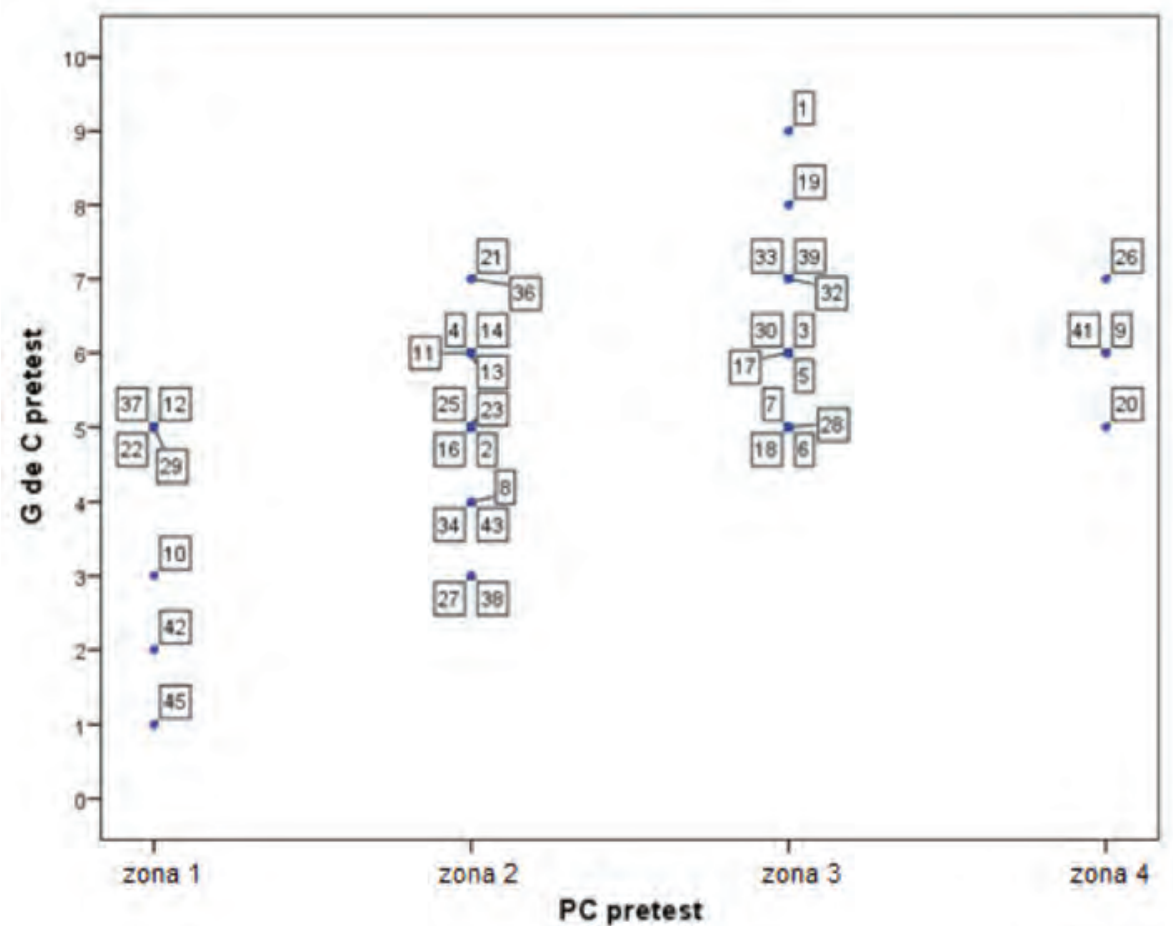

Figura 15: Grado de confianza de los alumnos en función del PCI o PC pre-test. 


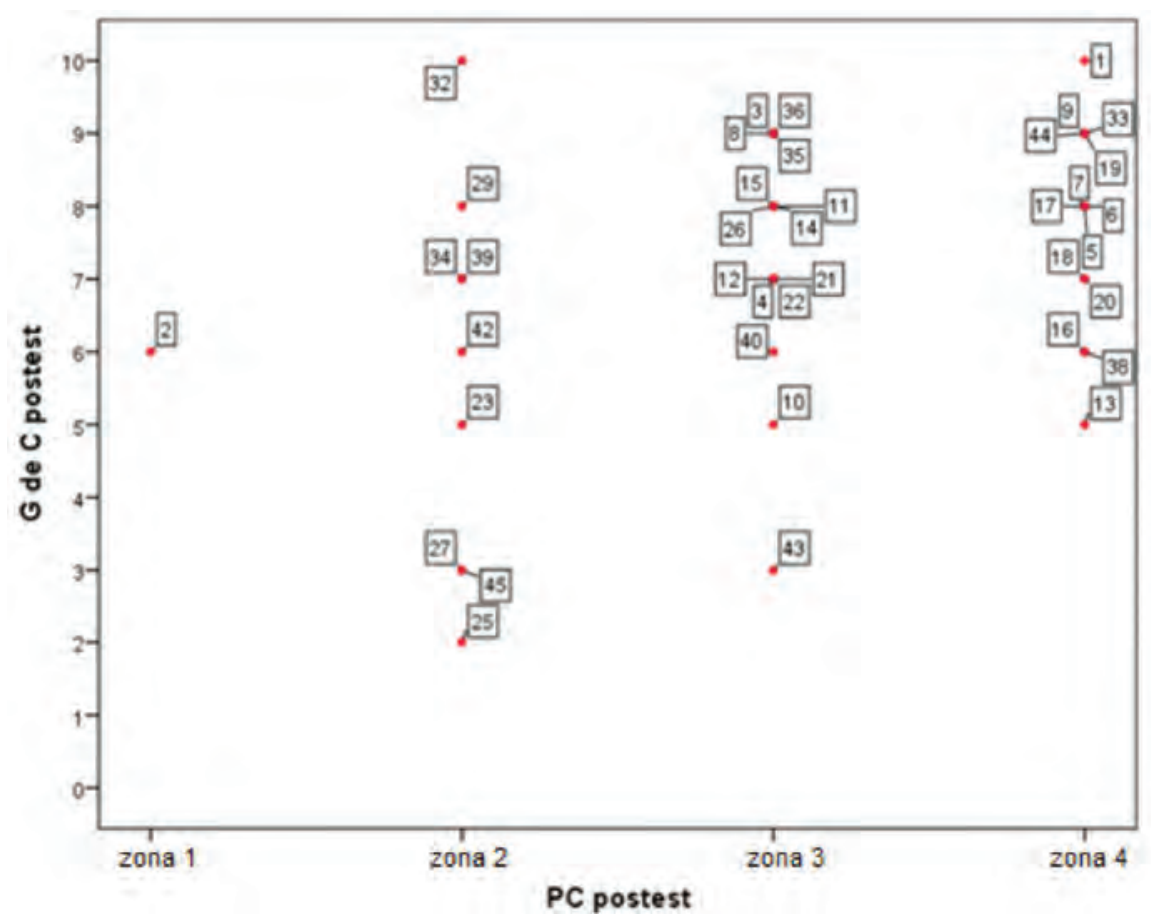

Figura 16: Grado de confianza de los alumnos en función del PCF o PC pos-test.

Con el objeto de comparar tanto los PC globales como los grados de confianza, se procedió al análisis estadístico para dos muestras relacionadas. Los resultados se indican en la Tabla 1.

Tabla 1: Prueba de los rangos con signos de Wilcoxon con los estadísticos de Prueba.

Prueba de los rangos con signos de Wilcoxon

\begin{tabular}{|c|c|c|c|c|}
\hline \multicolumn{5}{|c|}{ Rangos } \\
\hline & & $\mathbf{N}$ & Rango promedio & Suma de rangos \\
\hline \multirow{4}{*}{$\begin{array}{l}\text { PC global pos-test - PC } \\
\text { global pre-test }\end{array}$} & Rangos negativos & $6^{a}$ & 22,75 & 136,50 \\
\hline & Rangos positivos & $36^{b}$ & 21,29 & 766,50 \\
\hline & Empates & $2^{c}$ & & \\
\hline & Total & 44 & & \\
\hline \multirow{4}{*}{$\begin{array}{l}\mathrm{G} \text { de } \mathrm{C} \text { pos-test - } \mathrm{G} \text { de } \\
\mathrm{C} \text { pre-test }\end{array}$} & Rangos negativos & $3^{\mathrm{d}}$ & 14,00 & 42,00 \\
\hline & Rangos positivos & $37^{\mathrm{e}}$ & 21,03 & 778,00 \\
\hline & Empates & $4^{\mathrm{f}}$ & & \\
\hline & Total & 44 & & \\
\hline
\end{tabular}

Nota a: PC global pos-test $<$ PC global pre-test.

Nota b: PC global pos-test $>$ PC global pre-test.

Nota c: PC global pos-test $=$ PC global pre-test.

Nota d: G de C pos-test < G de C pre-test.

Nota e: $G$ de $C$ pos-test $>G$ de $C$ pre-test.

Nota f: $G$ de $C$ pos-test $=\mathrm{G}$ de $C$ pre-test. 


\section{Estadísticos de prueba ${ }^{a}$}

\begin{tabular}{lcc} 
& $\begin{array}{c}\text { PC global pos-test - PC global } \\
\text { pre-test }\end{array}$ & $\begin{array}{c}\text { G de C pos-test - G de C } \\
\text { pre-test }\end{array}$ \\
\hline$Z$ & $-3,945^{\mathrm{b}}$ & $-5,013^{\mathrm{b}}$ \\
\hline Sig. asintótica (bilateral) & 0,000 & 0,000 \\
\hline $\begin{array}{l}\text { Significación exacta (bilate- } \\
\text { ral) }\end{array}$ & 0,000 & 0,000 \\
\hline $\begin{array}{l}\text { Significación exacta (unila- } \\
\text { teral) }\end{array}$ & 0,000 & 0,000 \\
\hline Probabilidad en el punto & 0,000 & 0,000 \\
\hline
\end{tabular}

Nota a: Prueba de rangos con signo de Wilcoxon

Nota b: Se basa en rangos negativos.

\section{Prueba de los signos}

\begin{tabular}{|c|c|c|}
\hline \multicolumn{3}{|c|}{ Frecuencias } \\
\hline & & $\mathrm{N}$ \\
\hline \multirow{4}{*}{ PC global pos-test - PC global pre-test } & Diferencias negativas ${ }^{\mathrm{a}, \mathrm{d}}$ & 6 \\
\hline & Diferencias positivas ${ }^{\mathrm{b}, \mathrm{e}}$ & 36 \\
\hline & Empates ${ }^{\mathrm{c}, \mathrm{f}}$ & 2 \\
\hline & Total & 44 \\
\hline \multirow{4}{*}{$\mathrm{G}$ de $\mathrm{C}$ pos-test - $\mathrm{G}$ de $\mathrm{C}$ pre-test } & Diferencias negativas $^{\mathrm{a}, \mathrm{d}}$ & 3 \\
\hline & Diferencias positivas $^{\mathrm{b}, \mathrm{e}}$ & 37 \\
\hline & Empates $^{\mathrm{c}, \mathrm{f}}$ & 4 \\
\hline & Total & 44 \\
\hline
\end{tabular}

Nota a: PC global pos-test < PC global pre-test.

Nota b: PC global pos-test $>$ PC global pre-test.

Nota c: PC global pos-test $=$ PC global pre-test.

Nota d: $G$ de $C$ pos-test $<$ G de C pre-test.

Nota e: $G$ de $C$ pos-test $>\mathrm{G}$ de $C$ pre-test.

Nota f: $G$ de $C$ pos-test $=\mathrm{G}$ de $C$ pre-test.

\begin{tabular}{lcc} 
& Estadísticos de prueba $^{\mathbf{a}}$ \\
\hline & $\begin{array}{c}\text { PC global pos-test - PC } \\
\text { global pre-test }\end{array}$ & $\begin{array}{c}\text { G de C pos-test - G de C } \\
\text { pre-test }\end{array}$ \\
\hline$Z$ & $-4,475$ & $-5,218$ \\
\hline Sig. asintótica (bilateral) & 0,000 & 0,000 \\
\hline Significación exacta (bilateral) & 0,000 & 0,000 \\
\hline $\begin{array}{l}\text { Significación exacta (unila- } \\
\text { teral) }\end{array}$ & 0,000 & 0,000 \\
\hline Probabilidad en el punto & 0,000 & 0,000 \\
\hline
\end{tabular}

Nota a: Prueba de los signos.

Los resultados encontrados indican que se acepta $\mathrm{H}_{1}$, con un nivel de significación $\mathrm{p} \leq 0,05$. Es decir, los resultados obtenidos en el pre-test son distintos a los del pos-test. Si se observa con detalle, los rangos positivos son mayores a los negativos, por lo tanto, se distingue que los resultados del pos-test son superiores 
a los del pre-test. Expresado de otro modo, el pos-test indica un mayor nivel de PC y de Grado de Confianza respecto del pre-test, o una evolución en las zonas de PC globales del grupo indagado, mediante la intervención didáctica mediada por TIC.

La misma situación se observa para cada una de las categorías de análisis; si bien la excepción se da en la categoría No Conservación; es decir, no se presentaron diferencias significativas entre el examen pre-test y el pos-test debido a la intervención didáctica mediada por TIC en los procesos de No Conservación. Por razones de espacio, sólo se muestran las pruebas de Wilcoxon en el Anexo.

También en los gráficos que muestran las Figuras 17 y 18 se pueden observar dichos resultados. En las representaciones de PCF vs PCI, y de los grados de confianza en el pos-test vs en el pre-test, al trazar la diagonal en cada una de ellas, se advierte la densidad de alumnos en cada semi-área. En consecuencia, existen diferencias cualitativas que muestran que los resultados del pos-test son superiores al del pre-test en ambas categorías, si bien se evidencia que algunos estudiantes no muestran cambios, siendo éstos los ubicados sobre la diagonal.

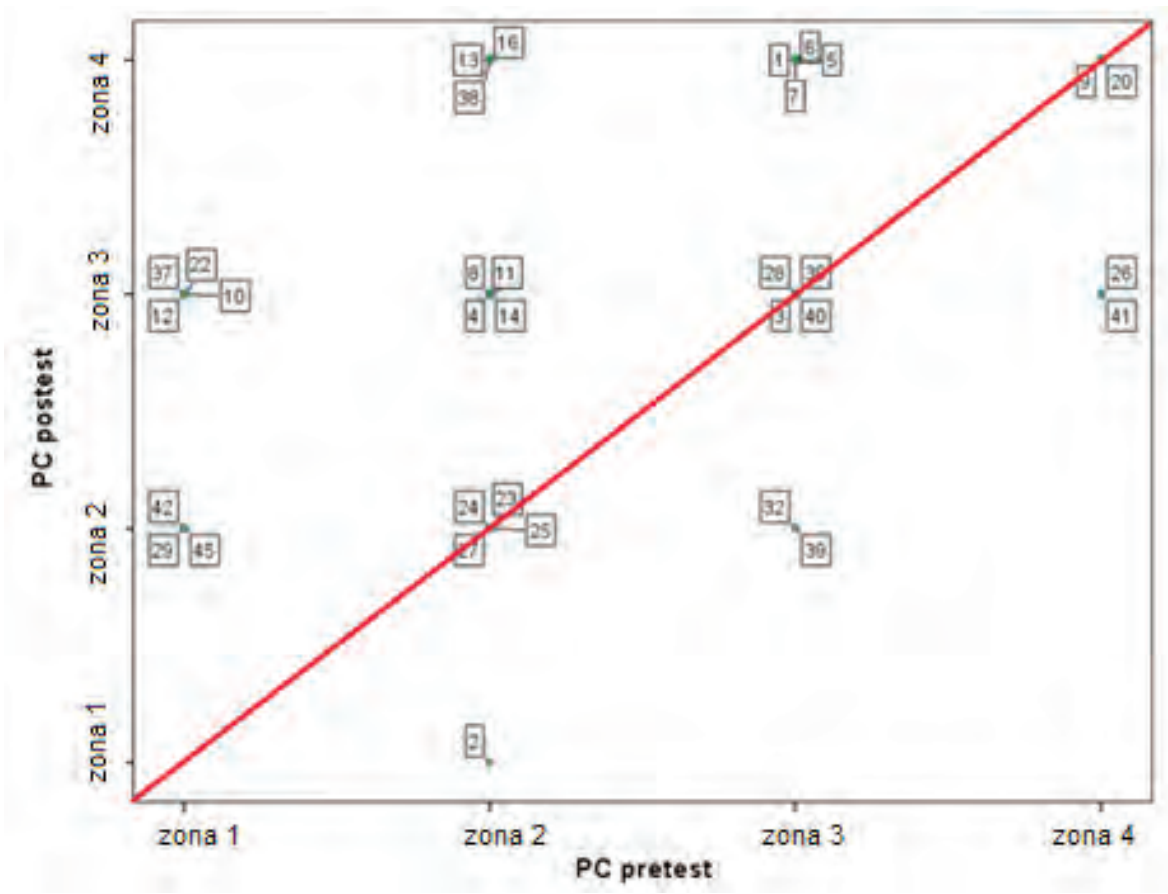

Figura 17: PC pos-test versus PC pre-test. 


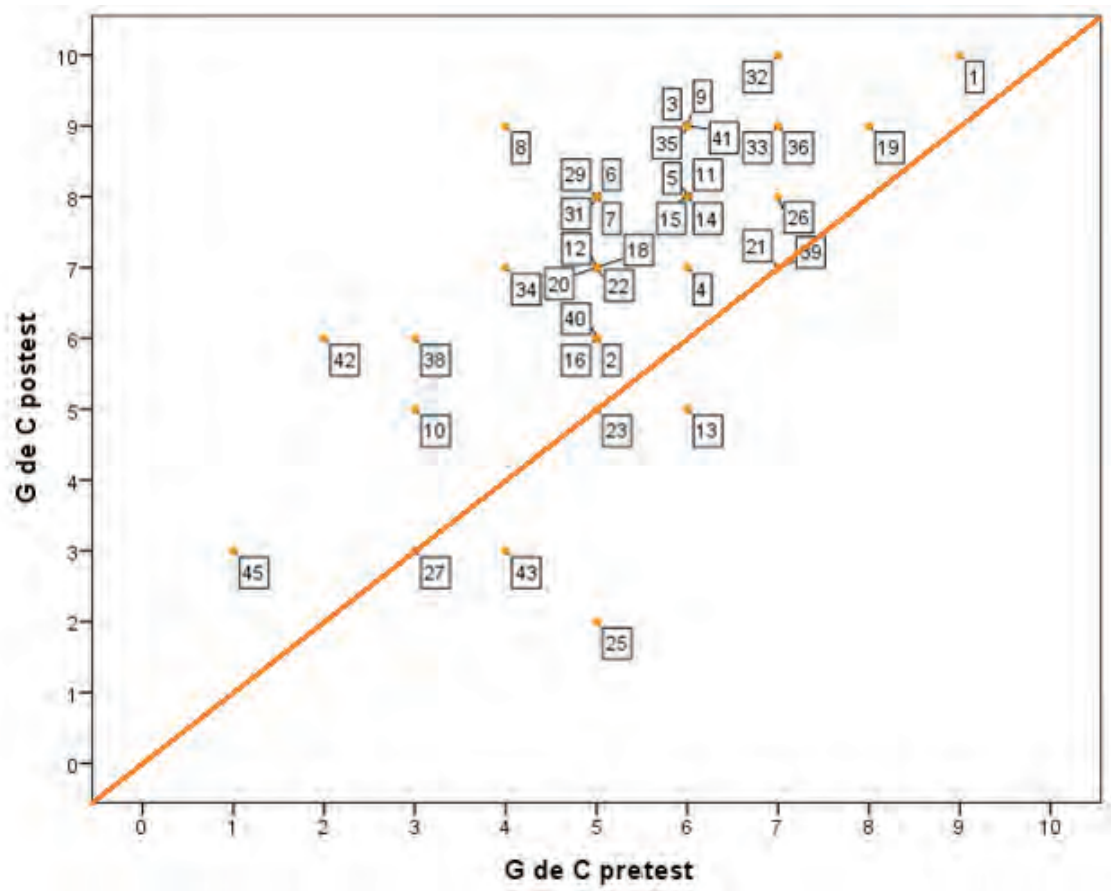

Figura 18. G de C pos-test versus pre-test.

\section{Conclusiones}

En este trabajo se presentó una nueva forma de evaluar una intervención didáctica asociada al estudio de los sistemas oscilantes, a través del empleo de Perfiles Conceptuales. Además, se planteó una modificación de dichos perfiles, incluyendo uno asociado a los procesos meta-cognitivos de los alumnos, denominado Perfil Conceptual Relativo (PCR).

La diferencia entre ambos tipos de perfiles permitió efectuar un análisis, tanto cualitativo como cuantitativo, acerca de los niveles de comprensión y apropiación de los conceptos científicos sobre los sistemas oscilantes, en alumnos de Ingeniería Civil que cursaron la materia Física I en el año 2017 en la UTN - FRSF.

Así, la incorporación de los PC propuestos por Mortimer como los Relativos a la meta-cognición formulados en esta investigación, permitió otra manera de evaluar el proceso de aprendizaje tanto de manera global, así como también por contenidos. Además fue posible analizar al grupo en su conjunto, o a cada sujeto individualmente.

Por lo tanto, el uso de PC muestra una enorme potencialidad acerca de las múltiples formas de indagar acerca de la comprensión de los Movimientos Oscilatorios.

Para finalizar, se considera que este análisis puede ser útil para el estudio y el análisis de cualquier contenido asociado a la Mecánica clásica o, en general, perteneciente a cualquier disciplina o ciencia. Es evidente que frente a las actuales evaluaciones consideradas "tradicionales", ésta es una metodología que puede llegar a suplantarlas con éxito. 


\section{Referencias bibliográficas}

Alonso, M., \& Finn, E. (1995). Física Vol. I, Mecánica. Wilmington, Delaware, EUA, Addison Wesley Iberoamericana.

Brotons, V., Baeza, F. J., Crespo Zaragoza, M. Á., \&Ivorra, S. (2014). Desarrollo de aplicaciones interactivas para la docencia de estructuras en Ingeniería Civil.

Camargo, É. P. D., Scalvi, L. V. D. A., \& Braga, T. M. S. (2007). Concepciones alternativas sobre reposo y movimiento, modelos históricos y deficiencia visual. Enseñanza de las ciencias, 25(2), 171-182.

Gil Pérez, D. \& Carrascosa Alís, J. (1992). Concepciones alternativas en mecánica. Dinámica: Las fuerzas como causa del movimiento. Selección de cuestiones elaboradas para su detección y tratamiento. Enseñanza de las Ciencias, 10(3), 314 - 328.

Clement, J. (1982). Student preconceptions in introductory mechanics. American Journal of Physics, $50(1), 66-71$.

Cohen, I. B. (1967). O nascimento de uma novafisica. Sáo Paulo: Livraria Editora.

Czudkova, L., \& Musilova, J. (2000). The pendulum: A stumbling block of secondary school mechanics. Physics Education, 35(6), 428.

de Lozano, S. R., Cardenas, S., \& Katz, V. (2016). Las concepciones no newtonianas en dinámica y su incidencia en el proceso de enseñanzaaprendizaje. Revista de Enseñanza de la Física, 2(2), 115-224.

El-Hani, C. N., \& Mortimer, E. F. (2010). O Valor Pragmático da Linguagem Cotidiana. Cunha, A. M.; Mortimer, E. F.; Aguiar Jr., O. G.; Nascimento, S. S., 327350.

Enrique, C. (2017). Conocimientos previos sobre movimiento oscilatorio de estudiantes de ingenierías. Saarbrücken: Editorial Académica Española.

Enrique, C.; Yanitelli, M. \& Giorgi, S. (2019). Un perfil conceptual para sistemas oscilantes en estudiantes de ingeniería. En:Beatriz Macedo, Sara Silveira, Daniel Meziat, Margarita García Astete, Luis Bengochea (Editores). $\mathrm{X}$ Congreso Iberoamericano de Educación Científica (Enseñanza y Aprendizaje de las Ciencias en Debate); Montevideo, Uruguay, 3, 101 110.

Enrique, C., Yanitelli, M., \& Giorgi, S. (2018). Dinámica de sistemas oscilantes: concepciones en estudiantes de ingeniería. Revista de Enseñanza de la Física, 30, 63-72.

Flavell, J. H. (1976). Metacognitive aspects of problema solving. The nature of intelligence, 231-235.

GarcíaBarneto, A. \& Bolivar Raya, J. P. (2008). Efecto de las simulaciones interactivas sobre las concepciones de los alumnos en relación con el movimiento armónico simple. Revista Electrónica de Enseñanza de las Ciencias, 7(3), 681-703.

García Barneto, A., \& Bolívar Raya, J. P. (2005). Uso de simulaciones informáticas en la enseñanza de la física: movimientos armónico simple y ondulatorio. Enseñanza de las ciencias, (Extra), 0001-5.

Herrera Espinosa, D. (2013). Ideas previas sobre el concepto de fuerza (Doctoral dissertation).

López, L. P. (2009). Buridán, el impetus y la primera unificación de la física terrestre y celeste. Thémata. Revista de Filosofía, (41). 
Manabu, S. (2004). The reproduction of scientific understanding about pendulum motion in the public. Science\&Education, 13(4-5), 473-492.

Mora, C., \& Benítez, Y. (2007). Errores conceptuales sobre fuerza y su impacto en la enseñanza. Revista cubana de física, 24(1), 41-45.

Mora, C., \& Herrera, D. (2009). Una revisión sobre ideas previas del concepto de fuerza. Latin-American Journal of PhysicsEducation, 3(1), 13.

Mortimer, E. (2000). Lenguaje y formación de conceptos en la enseñanza de las Ciencias. A. Machado Libros, Madrid (España).

Mortimer, E. F. (1995). Conceptual change or conceptual profile change? Science\& Education, 4(3), 267-285.

Mortimer, E. F., \& Carvalho, A. M. P. de. (1994). Evolução do atomismo em sala de aula: mudança de perfis conceituais. Universidade de São Paulo, São Paulo.

Peduzzi, L. O., \& Zylbersztajn, A. (1997). La física de la fuerza impresa y sus implicaciones para la enseñanza de la mecánica. Enseñanza de las ciencias: revista de investigación y experiencias didácticas, 15(3), 351-359; pp. 354.

Piaget, J., \& García, R. (1982). Psicogénesis e historia de la ciencia. Siglo Veintiuno. México.

Pozo, J. I. (1987). La historia se repite: las concepciones espontáneas sobre el movimiento y la gravedad. Infancia y aprendizaje, 10(38), 69-87.

Santos Benito, J. V., Martí, A. G., \& Selva, V. F. S. (2005). Recursos para la enseñanza del péndulo simple: imágenes, mediciones, simulaciones y guías didácticas. Caderno Brasileiro de Ensino de Física, 22(2), 165-189.

Solsona, N., Izquierdo, M., \& De Jong, O. (2001). Un estudio de la evolución de los perfiles conceptuales del alumnado sobre la reacción química/A study of evolution of student's knowledge abou tchem < icalreaction. Journal of Science Education, 2(1), 44.

Viennot, L. (1989). La didáctica en la enseñanza superior ’ para qué? Enseñanza de las ciencias: revista de investigación y experiencias didácticas, 7(1), 3-13.

Viennot, L. (1979). Spontaneous reasoning in elementary dynamics. European journal of science education, 1(2), 205-22

Viggiano, E., \& Rodrigues de Mattos, C. R. (2007). É possivel definir contextos de uso de zonas de perfil conceitual comum questionário? http://fep.if.usp.br/ profis/ arquivos/vienpec/CR2/p357.pdf (Consultado del 15 de agosto de 2017). 


\section{Anexo}

Análisis estadísticos de las comparaciones entre los PCI - pre-test - y PCF pos-test -; y entre los PCIR - pre-test - y PCFR - pos-test -.

\section{a. Conceptos Básicos}

\begin{tabular}{|c|c|c|c|c|}
\hline \multicolumn{5}{|c|}{ Rangos } \\
\hline & & $\mathbf{N}$ & Rango promedio & Suma de rangos \\
\hline \multirow{4}{*}{$\begin{array}{l}\text { PC Conceptos Basicos- } \\
\text { pos-test - PC Concep- } \\
\text { tos Basicospre-test }\end{array}$} & Rangos negativos & $8^{a}$ & 8,38 & 67,00 \\
\hline & Rangos positivos & $14^{\mathrm{b}}$ & 13,29 & 186,00 \\
\hline & Empates & $18^{c}$ & & \\
\hline & Total & 40 & & \\
\hline \multirow{4}{*}{$\begin{array}{l}\text { G de C Conceptos } \\
\text { Basicospos-test - G de } \\
\text { C Conceptos Basicos- } \\
\text { pre-test }\end{array}$} & Rangos negativos & $5^{\mathrm{d}}$ & 9,50 & 47,50 \\
\hline & Rangos positivos & $30^{e}$ & 19,42 & 582,50 \\
\hline & Empates & $5^{\mathrm{f}}$ & & \\
\hline & Total & 40 & & \\
\hline
\end{tabular}

Nota a: PC Conceptos Basicospos-test < PC Conceptos Basicospre-test.

Nota b: PC Conceptos Basicospos-test $>$ PC Conceptos Basicospre-test

Nota c: PC Conceptos Basicospos-test $=$ PC Conceptos Basicospre-test.

Nota d: G de C Conceptos Basicospos-test < G de C Conceptos Basicospre-test.

Nota e: G de C Conceptos Basicospos-test > G de C Conceptos Basicospre-test

Nota f: G de C Conceptos Basicospos-test = G de C Conceptos Basicospre-test

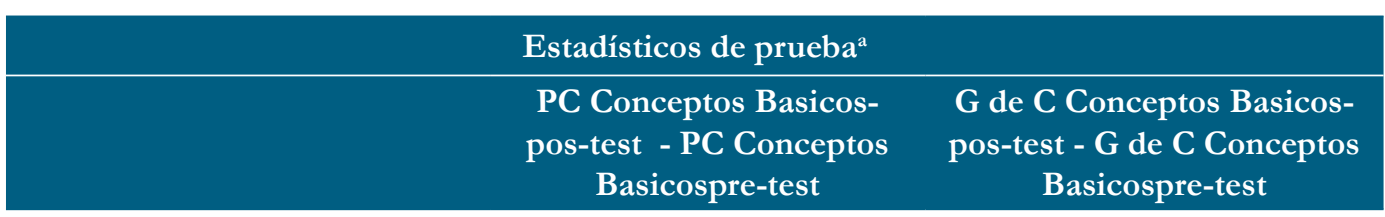

\begin{tabular}{lll} 
Significación exacta (bilateral) & $0,286^{\mathrm{b}}$ & 0,000 \\
\hline Significación exacta (unilateral) & 0,143 & 0,000 \\
\hline Probabilidad en el punto & 0,076 & 0,000 \\
\hline Z & & $-4,057$ \\
\hline Sig. asintótica (bilateral) & 0,000 \\
\hline
\end{tabular}

Nota a: Prueba de los signos

Nota b: Distribución binomial utilizada. 


\section{b. Cinemática}

\begin{tabular}{|c|c|c|c|c|}
\hline \multicolumn{5}{|c|}{ Rangos } \\
\hline & & $\mathbf{N}$ & Rango promedio & Suma de rangos \\
\hline \multirow{4}{*}{$\begin{array}{l}\text { PC Cinematica- } \\
\text { pos-test - PC Cinema- } \\
\text { ticapre-test }\end{array}$} & Rangos negativos & $5^{\mathrm{a}}$ & 8,50 & 42,50 \\
\hline & Rangos positivos & $14^{\mathrm{b}}$ & 10,54 & 147,50 \\
\hline & Empates & $22^{c}$ & & \\
\hline & Total & 41 & & \\
\hline \multirow{4}{*}{$\begin{array}{l}\text { G de C Cinematica- } \\
\text { pos-test - G de C PC } \\
\text { Cinematicapre-test }\end{array}$} & Rangos negativos & $6^{\mathrm{d}}$ & 15,08 & 90,50 \\
\hline & Rangos positivos & $24^{\mathrm{e}}$ & 15,60 & 374,50 \\
\hline & Empates & $11^{\mathrm{f}}$ & & \\
\hline & Total & 41 & & \\
\hline
\end{tabular}

Nota a: PC Conceptos Basicospos-test < PC Conceptos Basicospre-test.

Nota b: PC Conceptos Basicospos-test $>$ PC Conceptos Basicospre-test

Nota c: PC Conceptos Basicospos-test $=$ PC Conceptos Basicospre-test.

Nota d: G de C Conceptos Basicospos-test < G de C Conceptos Basicospre-test.

Nota e: $G$ de C Conceptos Basicospos-test $>$ G de C Conceptos Basicospre-test

Nota f: G de C Conceptos Basicospos-test = G de C Conceptos Basicospre-test

\begin{tabular}{lcc}
\multicolumn{3}{c}{ Estadísticos de prueba $^{\mathrm{a}}$} \\
& $\begin{array}{c}\text { PC Cinematicapos-test - } \\
\text { PC Cinematicapre-test }\end{array}$ & $\begin{array}{c}\text { G de C Cinematicapos-test - G } \\
\text { de C PC Cinematicapre-test }\end{array}$ \\
\hline$Z$ & $-2,275^{\mathrm{b}}$ & $-2,963^{\mathrm{b}}$ \\
\hline Sig. asintótica (bilateral) & 0,023 & 0,003 \\
\hline Significación exacta (bilateral) & 0,028 & 0,002 \\
\hline Significación exacta (unilateral) & 0,014 & 0,001 \\
\hline Probabilidad en el punto & 0,008 & 0,000 \\
\hline
\end{tabular}

Nota a: Prueba de rangos con signo de Wilcoxon.

Nota b: Se basa en rangos negativos.. 


\section{c. Dinámica}

\begin{tabular}{lllcc} 
& \multicolumn{4}{c}{ Rangos } \\
& & N & Rango promedio & Suma de rangos \\
& Rangos negativos & $4^{\mathrm{a}}$ & 10,50 & 42,00 \\
\cline { 2 - 5 } $\begin{array}{l}\text { PC Dinamicapos-test }- \\
\text { PC Dinamicapre-test }\end{array}$ & Rangos positivos & $18^{\mathrm{b}}$ & 11,72 & 211,00 \\
\cline { 2 - 5 } & Empates & $22^{\mathrm{c}}$ & & \\
\cline { 2 - 5 } & Total & 44 & & 30,50 \\
\hline \multirow{3}{*}{$\begin{array}{l}\text { G de C Dinamica- } \\
\text { pos-test - G de C Dina- } \\
\text { micapre-test }\end{array}$} & Rangos negativos & $3^{\mathrm{d}}$ & 10,17 & 789,50 \\
\cline { 2 - 5 } & Rangos positivos & $37^{\mathrm{e}}$ & 21,34 & \\
\cline { 2 - 5 } & Empates & $4^{\mathrm{f}}$ & & \\
\cline { 2 - 5 } & Total & 44 & & \\
\hline
\end{tabular}

Nota a: PC Dinamicapos-test $<$ PC Dinamicapre-test

Nota b: PC Dinamicapos-test $>$ PC Dinamicapre-test

Nota c: PC PC Dinamicapos-test $=$ PC Dinamicapre-test

Nota d: G de C Dinamicapos-test < G de C Dinamicapre-test

Nota e: G de C Dinamicapos-test $>$ G de C Dinamicapre-test

Nota f: $G$ de C Dinamicapos-test $=\mathrm{G}$ de C Dinamicapre-test

\begin{tabular}{lcc} 
& Estadísticos de prueba $^{\mathrm{a}}$ & \\
& $\begin{array}{c}\text { PC Dinamicapos-test - PC } \\
\text { Dinamicapre-test }\end{array}$ & $\begin{array}{c}\text { G de C Dinamicapos-test - } \\
\text { G de C Dinamicapre-test }\end{array}$ \\
\hline$Z$ & $-3,021^{\mathrm{b}}$ & $-5,145^{\mathrm{b}}$ \\
\hline Sig. asintótica (bilateral) & 0,003 & 0,000 \\
\hline Significación exacta (bilateral) & 0,003 & 0,000 \\
\hline Significación exacta (unilateral) & 0,001 & 0,000 \\
\hline Probabilidad en el punto & 0,001 & 0,000 \\
\hline
\end{tabular}

Nota a: Prueba de rangos con signo de Wilcoxon

Nota b: Se basa en rangos negativos. 


\section{d. Energía}

\begin{tabular}{|c|c|c|c|c|}
\hline \multicolumn{5}{|c|}{ Rangos } \\
\hline & & $\mathbf{N}$ & Rango promedio & Suma de rangos \\
\hline \multirow{4}{*}{$\begin{array}{l}\text { PC Energia - pos-test - } \\
\text { PC Energía - pre-test }\end{array}$} & Rangos negativos & $5^{\mathrm{a}}$ & 15,50 & 77,50 \\
\hline & Rangos positivos & $25^{\mathrm{b}}$ & 15,50 & 387,50 \\
\hline & Empates & $10^{c}$ & & \\
\hline & Total & 40 & & \\
\hline \multirow{4}{*}{$\begin{array}{l}\text { G de C Energía - pos- } \\
\text { test - G de C Energía } \\
\text { - pre-test }\end{array}$} & Rangos negativos & $\mathrm{O}^{\mathrm{d}}$ & 0,00 & 0,00 \\
\hline & Rangos positivos & $40^{e}$ & 20,50 & 820,00 \\
\hline & Empates & $o^{f}$ & & \\
\hline & Total & 40 & & \\
\hline
\end{tabular}

Nota a: PC Energia - pos-test < PC Energía - pre-test

Nota b: PC Energia - pos-test $>$ PC Energía - pre-test

Nota c: PC Energia - pos-test $=$ PC Energía - pre-test

Nota d: G de C Energía - pos-test < G de C Energía - pre-test

Nota e: G de C Energía - pos-test $>$ G de C Energía - pre-test

Nota f: $G$ de $C$ Energía - pos-test $=G$ de $C$ Energía -pre-test

\begin{tabular}{|c|c|c|}
\hline \multicolumn{3}{|c|}{ Estadísticos de prueba ${ }^{a}$} \\
\hline & $\begin{array}{c}\text { PC Energia - pos-test - PC } \\
\text { Energía - pre-test }\end{array}$ & $\begin{array}{l}\text { G de C Energía - pos-test - } \\
\text { G de C Energía - pre-test }\end{array}$ \\
\hline $\mathrm{Z}$ & $--3,197^{b}$ & $-5,517^{b}$ \\
\hline Sig. asintótica (bilateral) & 0,001 & 0,000 \\
\hline Significación exacta (bilateral) & 0,001 & 0,000 \\
\hline Significación exacta (unilateral) & 0,000 & 0,000 \\
\hline Probabilidad en el punto & 0,000 & 0,000 \\
\hline
\end{tabular}

Nota a: Prueba de rangos con signo de Wilcoxon

Nota b: Se basa en rangos negativos. 


\section{d. Conservación}

\begin{tabular}{|c|c|c|c|c|}
\hline \multicolumn{5}{|c|}{ Rangos } \\
\hline & & $\mathbf{N}$ & Rango promedio & Suma de rangos \\
\hline \multirow{4}{*}{$\begin{array}{l}\text { PC Conserva- } \\
\text { cionpos-test - PC Con- } \\
\text { servacionpre-test }\end{array}$} & Rangos negativos & $5^{a}$ & 10,00 & 50,00 \\
\hline & Rangos positivos & $19^{\mathrm{b}}$ & 13,16 & 250,00 \\
\hline & Empates & $16^{c}$ & & \\
\hline & Total & 40 & & \\
\hline \multirow{4}{*}{$\begin{array}{l}\text { G de C Conserva- } \\
\text { cionpos-test - G de C } \\
\text { Conservacionpre-test }\end{array}$} & Rangos negativos & $\mathrm{O}^{\mathrm{d}}$ & 0,00 & 0,00 \\
\hline & Rangos positivos & $40^{e}$ & 20,50 & 820,00 \\
\hline & Empates & $\mathrm{O}^{\mathrm{f}}$ & & \\
\hline & Total & 40 & & \\
\hline
\end{tabular}

Nota a: PC Conservacionpos-test $<$ PC Conservacionpre-test

Nota b: PC Conservacionpos-test $>$ PC Conservacionpre-test

Nota c: PC Conservacionpos-test $=$ PC Conservacionpre-test

Nota d: G de C Conservacionpos-test < G de C Conservacionpre-test

Nota e: $G$ de C Conservacionpos-test $>$ G de C Conservacionpre-test

Nota f: $\mathrm{G}$ de $\mathrm{C}$ Conservacionpos-test $=\mathrm{G}$ de $\mathrm{C}$ Conservacionpre-test

\begin{tabular}{lcc} 
& Estadísticos de prueba $^{\mathrm{a}}$ & \\
\hline & $\begin{array}{c}\text { PC Conservacionpos-test - } \\
\text { PC Conservacionpre-test }\end{array}$ & $\begin{array}{c}\text { G de C Conserva- } \\
\text { cionpos-test - G de C } \\
\text { Conservacionpre-test }\end{array}$ \\
\hline$Z$ & $-3,043^{\mathrm{b}}$ & $-5,559^{\mathrm{b}}$ \\
\hline Sig. asintótica (bilateral) & 0,002 & 0,000 \\
\hline Significación exacta (bilateral) & 0,002 & 0,000 \\
\hline Significación exacta (unilateral) & 0,001 & 0,000 \\
\hline Probabilidad en el punto & 0,001 & 0,000
\end{tabular}

Nota a: Prueba de rangos con signo de Wilcoxon

Nota b: Se basa en rangos negativos. 


\section{f. No Conservación}

\begin{tabular}{|c|c|c|c|c|}
\hline \multicolumn{5}{|c|}{ Rangos } \\
\hline & & $\mathbf{N}$ & Rango promedio & Suma de rangos \\
\hline \multirow{4}{*}{$\begin{array}{l}\text { PC No Conserva- } \\
\text { cionpos-test - PC No } \\
\text { Conservacionpre-test }\end{array}$} & Rangos negativos & $10^{a}$ & 12,20 & 122,00 \\
\hline & Rangos positivos & $12^{\mathrm{b}}$ & 10,92 & 131,00 \\
\hline & Empates & $14^{\mathrm{c}}$ & & \\
\hline & Total & 36 & & \\
\hline \multirow{4}{*}{$\begin{array}{c}\text { G de C No Conser- } \\
\text { vacionpos-test - G } \\
\text { de C No Conserva- } \\
\text { cionpre-test }\end{array}$} & Rangos negativos & $4^{\mathrm{d}}$ & 6,88 & 27,50 \\
\hline & Rangos positivos & $24^{\mathrm{e}}$ & 15,77 & 378,50 \\
\hline & Empates & $8^{f}$ & & \\
\hline & Total & 36 & & \\
\hline
\end{tabular}

Nota a: PC No Conservacionpos-test $<$ PC No Conservacionpre-test

Nota b: PC No Conservacionpos-test $>$ PC No Conservacionpre-test

Nota c: PC No Conservacionpos-test $=$ PC No Conservacionpre-test

Nota d: G de C No Conservacionpos-test $<$ G de C No Conservacionpre-test

Nota e: G de C No Conservacionpos-test $>$ G de C No Conservacionpre-test

Nota f: $\mathrm{G}$ de $\mathrm{C}$ No Conservacionpos-test $=\mathrm{G}$ de $\mathrm{C}$ No Conservacionpre-test

\begin{tabular}{lcc}
\multicolumn{3}{c}{ Estadísticos de prueba $^{\mathrm{a}}$} \\
& $\begin{array}{c}\text { PC Conservacionpos-test - } \\
\text { PC Conservacionpre-test }\end{array}$ & $\begin{array}{c}\text { G de C Conservacionpos-test } \\
\text { - G de C Conserva- } \\
\text { cionpre-test }\end{array}$ \\
\hline$Z$ &,$- 152^{\mathrm{b}}$ & $-4,038^{\mathrm{b}}$ \\
\hline Sig. asintótica (bilateral) & 0,879 & 0,000 \\
\hline Significación exacta (bilateral) & 0,892 & 0,000 \\
\hline Significación exacta (unilateral) & 0,446 & 0,000 \\
\hline Probabilidad en el punto & 0,018 & 0,000 \\
\hline
\end{tabular}

Nota a: Prueba de rangos con signo de Wilcoxon

Nota b: Se basa en rangos negativos. 